\title{
Podocytes and microRNA-30/Cx43 axis in diabetic nephropathy
}

\author{
Seo Rin Kim ${ }^{1}$, Soon Hyo Kwon ${ }^{2}$ \\ ${ }^{1}$ Department of Nephrology, Pusan National University Yangsan Hospital, Yangsan, Korea; ${ }^{2}$ Division of Nephrology, Soonchunhyang University \\ Seoul Hospital, Seoul, Korea \\ Correspondence to: Soon Hyo Kwon, MD, PhD. Division of Nephrology, Soonchunhyang University Seoul Hospital, 59 Daesagwan-ro, Yongsan-gu, \\ Seoul 04401, Korea. Email: ksoonhyo@schmc.ac.kr.
}

Submitted Mar 05, 2021. Accepted for publication Mar 28, 2021.

doi: $10.21037 / \mathrm{atm}-21-1036$

View this article at: http://dx.doi.org/10.21037/atm-21-1036

MicroRNAs are small non-coding (nc) RNAs (20-25 nucleotide long structures) that bind to the 3 '-untranslated region of target messenger RNAs and block their translation, leading to gene silencing at the posttranscriptional level (1). MicroRNAs potentially regulate $60 \%$ of protein-coding genes in humans (1). Since the report of microRNA-192 as the first specific microRNA in diabetic nephropathy, the role of various microRNAs in its pathogenesis has been studied extensively over the past few decades (2). The functions of individual microRNAs in diabetic nephropathy could provide clues for new therapeutic targets. Further, imbalance of microRNA expression, either upregulation or downregulation, contributes to the progression and prognosis of diabetic nephropathy by modulating genes related to ECM accumulation, hypertrophy, oxidative stress, inflammation, endoplasmic reticulum stress (ERS), and signal transduction in a variety of renal cells (1). Upregulated microRNAs including miR-21, miR-34a-5p, miR-141, and miR-370 have been investigated in diabetic nephropathy (3). Among these, miR-2 1 is one of the most studied microRNAs. miR21 induces renal injury and fibrosis by promoting cell cycle impairment and mesangial hypertrophy, and by activating TGF- $\beta 1$ and Akt signaling via downregulation of SMAD7 and PTEN, respectively (2). In contrast, the expression of some microRNAs including the Let-7 family, miR-25, miR29 family, miR-93, miR-126, miR-130b, miR-424, and miR146a is decreased in diabetic nephropathy (3). Low levels of miR-25 in the blood of diabetic patients are associated with enhanced oxidative stress. The miR-29 family is regarded as a mediator of TGF- $\beta 1$-mediated fibrogenesis. In addition to miR-29, miR-146a is known as an anti-inflammatory microRNA and is decreased in diabetic nephropathy (2).
Thus, correcting microRNA imbalance is a potential therapeutic strategy for this disease.

In the progression of diabetic nephropathy, loss of podocytes is considered an early and pivotal mechanism (4). The microRNA-30 family including microRNA-30a through microRNA-30e, is abundantly expressed in podocytes, and plays an important role in controlling podocyte homeostasis (5). Connexins are components of intercellular hemichannels, connexons, which form gap junctions and allow cell-to-cell communication. Connexin $43(\mathrm{Cx} 43)$ is considered the most widely expressed and distributed gap junction protein related to gene transcription, cytoskeletal dynamics, ATP exocytosis, vesicle release, and cell stress (6).

Recently, Li et al. (7) reported the role of microRNA-30/ $\mathrm{Cx} 43$ in podocyte injury in diabetic nephropathy. They found that microRNA-30 members were noticeably downregulated in high glucose-stimulated podocytes and streptozotocin-induced diabetic nephropathy model rats. They further identified $\mathrm{Cx} 43$ as the potential target of the microRNA-30 family using TargetScan and proved their functional interaction using a luciferase reporter assay. The authors subsequently applied a microRNA-30 family mimic and inhibitor both in vitro and in vivo and $\mathrm{Cx} 43$ siRNA in vitro, demonstrating that overexpression of microRNA-30 members and inhibition of $\mathrm{Cx} 43$ prevent podocyte injury. To further investigate the mechanisms of the microRNA-30/Cx43 axis in podocyte apoptosis, the authors focused on ERS showing concurrent changes and co-localization of caspase 12 and Cx43. Finally, they found that overexpression of microRNA-30 family members attenuated ERS by modulating $\mathrm{Cx} 43$ expression.

The microRNA-30 family may be involved in podocyte 


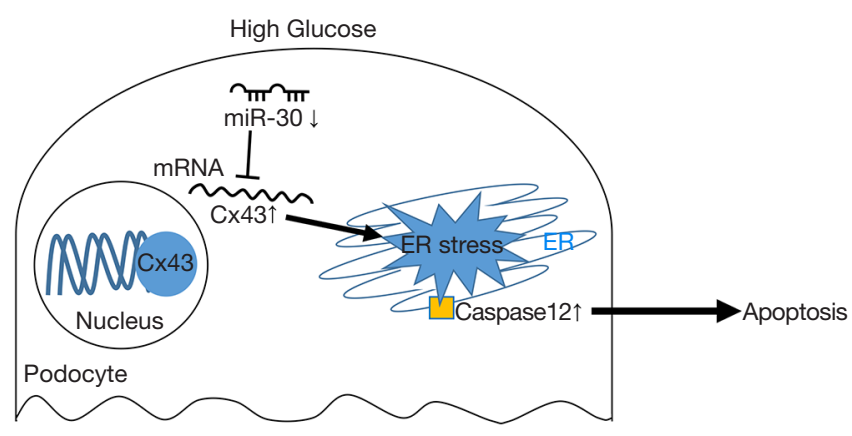

Figure 1 MicroRNA-30/Cx43/ERS axis in podocyte injury during diabetic nephropathy. $\mathrm{Cx} 43$, connexin-43; ERS, endoplasmic reticulum stress; mRNA, messenger RNA; miR, microRNA.

injury through diverse pathways. It inhibits Notch 1 activation and $\mathrm{p} 53$ signaling pathways, as well as cytoskeletal damage (5). microRNA-30 members have also been reported to regulate calcium/calcineurin signaling, and microRNA-30a is known to inhibit epithelial-mesenchymal transition in podocytes $(2,8)$. Metaherin is another target of microRNA-30 members, which leads to podocyte apoptosis in diabetic nephropathy by activating the p38 MAPK signaling pathway (9). According to Li et al., all five members of the microRNA-30 family significantly changed in the same direction in diabetic nephropathy. The microRNA-30 family is known to be encoded by six distinct genes located in discrete locations on the chromosomes (5). Consequently, the damaging factors in diabetic nephropathy may be common signals that promote all these genes.

The expression of $\mathrm{Cx} 43$ in diabetic kidneys appears to be diverse depending on the species and types of renal cells. Renal expression of $\mathrm{Cx} 43$ was found to be downregulated in a diabetic rat model (10) as well as in diabetic patients without specifying the cell types affected (11). However, another study using SD rats reported that podocyte injury was related to increased expression of $\mathrm{Cx} 43$ through impaired autophagic flux (12). In rodents, Cx43 localization is sparsely observed along the glomerular capillary wall in normal glomeruli, but becomes apparent only after podocyte injury (13). In humans, Cx43 is most abundantly expressed in podocytes with a linear pattern, but diabetic nephropathy downregulates and redistributes the $\mathrm{Cx} 43$ expression (14). Thus, whether the effect of the microRNA-30/Cx43 axis is conserved in the human kidney, remains to be determined.

The authors also studied ERS-induced podocyte apoptosis in diabetic nephropathy. In this study, the authors found increased expression of caspase-12, a key molecule implicated in ERS-induced apoptosis; however, caspase-12 alone might be insufficient to explain ERS. Nevertheless, the authors showed that treatment with microRNA-30 members mimics and Cx43 siRNA effectively restored podocyte apoptosis and the expression of caspase-12, supporting the relationship between microRNA-30/Cx43 and ERS (Figure 1).

Although the authors elicited the bottom line, they were inclined to simplify the complicated mechanisms of podocyte apoptosis and ERS in terms of methodology. Further studies considering diverse indicators and aspects are thus needed to better understand the microRNA-30/ Cx43/ER axis in diabetic nephropathy. Nevertheless, the authors revealed novel functional connections among microRNA-30 members, $\mathrm{Cx}_{x} 43$, and ERS leading to podocyte injury in diabetic nephropathy. In future clinical applications of this axis as a therapeutic target, testing a single microRNA as a biomarker or therapeutic target should be done cautiously, considering the interactions of various microRNAs.

In summary, the study by Li et al. highlighted the effect of the microRNA-30/Cx43 axis on podocyte injury in diabetic nephropathy. The authors provide insights into podocyte apoptosis in terms of microRNA-30 members and their targets. Despite the need for extensive research on the interactions between various microRNAs and pathological signaling pathways, the microRNA-30/Cx43/ERS axis may be a potential therapeutic target in diabetic nephropathy.

\section{Acknowledgments}

Funding: This work was supported by the Soonchunhyang Research Fund.

\section{Footnote}

Provenance and Peer Review: This article was commissioned by the editorial office, Annals of Translational Medicine. The article did not undergo external peer review.

Conflicts of Interest: Both authors have completed the ICMJE uniform disclosure form (available at http://dx.doi. org/10.21037/atm-21-1036). Dr. SHK reports grants from Soonchunhyang Research Fund during the conduct of the study, and Korean National Research Fund (NRF2020R1I1A3A04037367) outside the submitted work. The 
other author has no conflicts of interest to declare.

Ethical Statement: The authors are accountable for all aspects of the work in ensuring that questions related to the accuracy or integrity of any part of the work are appropriately investigated and resolved.

Open Access Statement: This is an Open Access article distributed in accordance with the Creative Commons Attribution-NonCommercial-NoDerivs 4.0 International License (CC BY-NC-ND 4.0), which permits the noncommercial replication and distribution of the article with the strict proviso that no changes or edits are made and the original work is properly cited (including links to both the formal publication through the relevant DOI and the license). See: https://creativecommons.org/licenses/by-nc-nd/4.0/.

\section{References}

1. Kato M. Noncoding RNAs as therapeutic targets in early stage diabetic kidney disease. Kidney Res Clin Pract 2018;37:197-209.

2. Sankrityayan H, Kulkarni YA, Gaikwad AB. Diabetic nephropathy: the regulatory interplay between epigenetics and microRNAs. Pharmacol Res 2019;141:574-85.

3. Tang J, Yao D, Yan H, et al. The role of microRNAs in the pathogenesis of diabetic nephropathy. Int J Endocrinol 2019;2019:8719060.

4. Alpers CE, Hudkins KL. Pathology identifies glomerular treatment targets in diabetic nephropathy. Kidney Res Clin Pract 2018;37:106-11.

5. Wu J, Zheng C, Fan Y, et al. Downregulation of

Cite this article as: Kim SR, Kwon SH. Podocytes and microRNA-30/Cx43 axis in diabetic nephropathy. Ann Transl Med 2021;9(10):828. doi: 10.21037/atm-21-1036
microRNA-30 facilitates podocyte injury and is prevented by glucocorticoids. J Am Soc Nephrol 2014;25:92-104.

6. Prakoura N, Kavvadas P, Chadjichristos CE. Connexin 43: a new therapeutic target against chronic kidney disease. Cell Physiol Biochem 2018;49:985.

7. Li M, Ni W, Zhang M, et al. MicroRNA-30/Cx43 axis contributes to podocyte injury by regulating ER stress in diabetic nephropathy. Ann Transl Med 2020;8:1674.

8. Wu J, Zheng C, Wang X, et al. MicroRNA-30 family members regulate calcium/calcineurin signaling in podocytes. J Clin Invest 2015;125:4091-106.

9. Liu WT, Peng FF, Li HY, et al. Metadherin facilitates podocyte apoptosis in diabetic nephropathy. Cell Death Dis 2016;7:e2477.

10. Zeng O, Li F, Li Y, et al. Effect of Novel Gasotransmitter hydrogen sulfide on renal fibrosis and connexins expression in diabetic rats. Bioengineered 2016;7:314-20.

11. Guo YN, Wang JC, Cai GY, et al. AMPK-mediated downregulation of connexin 43 and premature senescence of mesangial cells under high-glucose conditions. Exp Gerontol 2014;51:71-81.

12. Ji J, Zhao Y, Na C, et al. Connexin 43-autophagy loop in the podocyte injury of diabetic nephropathy. Int J Mol Med 2019;44:1781-8.

13. Yaoita E, Yao J, Yoshida Y, et al. Up-regulation of connexin43 in glomerular podocytes in response to injury. Am J Pathol 2002;161:1597-606.

14. Sawai K, Mukoyama M, Mori K, et al. Redistribution of connexin43 expression in glomerular podocytes predicts poor renal prognosis in patients with type 2 diabetes and overt nephropathy. Nephrol Dial Transplant 2006;21:2472-7. 